\title{
ERRATUM
}

\section{Retracted: Comparative Proteomic Analysis of Mesenchymal Stem Cells Derived from Human Bone Marrow, Umbilical Cord and Placenta: Implication in the Migration}

\author{
Guo Li, Xiao-Ai Zhang, Hua Wang, Xin Wang, Chun-Ling Meng, \\ Chu-Yan Chan, David Tai Wai Yew, Kam Sze Tsang, Karen Li, \\ Sau-na Tsai, Sai-Ming Ngai, Zhong Chao Han, \\ Marie Chia-Mi Lin, Ming-Liang He, and Hsiang-Fu Kung
}

\section{H.-F. Kung ( $\square)$}

Stanley Ho Centre for Emerging Infectious Diseases, The Chinese University of Hong Kong, Hong Kong, People's Republic of China

State Key Laboratory in Oncology in South China, Sun Yat-Sen University Cancer Center, Guangzhou, People's Republic of China e-mail: b110473@mailserv.cuhk.edu.hk

DOI 10.1007/978-1-4614-0254-1_18

Retraction: Chapter 5 "Comparative Proteomic Analysis of Mesenchymal Stem Cells Derived from Human Bone Marrow, Umbilical Cord and Placenta: Implication in the Migration" pages 51-68; duplicate publication. 